\title{
A Case of Metastatic Breast Cancer to the Meninges
}

\author{
Carolyn M. Ross, MSIII and Jie Cui, MD
}

\section{Case Report}

A 44-year-old woman with a history of triple-negative breast cancer metastatic to the brain presented with changes in mental status, lower back pain, and a left facial Bell's palsy.

The patient had previously been ambulatory and fully cognizant of her surroundings until one week prior to presentation when she began to exhibit progressively worsening confusion. As her mental status began to change, the patient developed new onset pain over her lumbar spine, supra-pubic area, bilateral flanks, hips, and knees. The pain was refractory to an epidural injection into her lumbar spine performed at the patient's local hospital. Other complaints included a recent onset urinary retention, weakness of the left lower extremity, a left facial Bell's palsy, and difficulty walking. On admission, the patient was taking gabapentin, citalopram, aspirin, enoxaparin, nystatin, lopressor, docusate, lorazepam, and methylprednisone. The patient was treating her pain with hydromorphone hydrochloride as needed. No other medications had recently been prescribed.

Past medical history included breast cancer status post bilateral mastectomies, four cycles of chemotherapy with paclitaxel, and neo-adjuvant radiation. One month prior to presentation, the patient was found to have metastases to her brain, and underwent a left fronto-temporal craniotomy that was then followed by a course of whole brain irradiation.

Upon examination, the patient was confused and had difficulty speaking and swallowing. Her temperature was $97.4^{\circ} \mathrm{F}$, her pulse was 95 beats per minute, her respiratory rate was 20 breaths per minute, and her blood pressure was 143/88 mm Hg. The patient's pulse oximetry on room air was $95 \%$. Her pupils were anicteric and equally round and reactive to light, but a left visual field defect was noted. The patient exhibited facial nerve palsy involving the entire left side of the face. All other cranial nerves appeared intact. The patient showed diffuse myoclonic jerks in her upper extremities. Motor testing revealed 3/5 strength in the left leg and $4 / 5$ strength in the right leg. Sensation was preserved throughout the extremities. The patient's cardiac exam was normal, and her lungs were clear to auscultation bilaterally. Her abdomen was soft and non-distended, and her legs were non-edematous.

The patient's white blood cell count was 6300 cells $/ \mathrm{mm}^{3}$ with a differential of $76.3 \%$ neutrophils and $11.9 \%$ lymphocytes. Hemoglobin was $13.5 \mathrm{~g} / \mathrm{dL}$, hematocrit was $40.4 \%$, and platelets were $229,000 / \mathrm{ml}$. Electrolyte values and coagulation tests were both within normal limits. The patient's ESR was elevated at 60. Her liver function tests showed a total protein of $6.9 \mathrm{gm} /$ $\mathrm{dl}$, albumin of $4.2 \mathrm{gm} / \mathrm{dl}$, total bilirubin $1.4 \mathrm{mg} / \mathrm{dl}$, direct bilirubin $0.6 \mathrm{mg} / \mathrm{dl}$, AST of $33 \mathrm{U} / \mathrm{L}$, ALT $32 \mathrm{U} / \mathrm{L}$, and alkaline phosphatase of $82 \mathrm{U} / \mathrm{L}$. Blood and urine cultures were negative for bacterial growth.
A CT scan of the patient's spine revealed mild multilevel degenerative changes with no evidence of lytic or blastic lesions. An MRI of the spine showed no evidence of bony metastases, spinal cord compression, or significant spinal stenosis, and a long bone scan was also negative for any bone lesions. A head CT scan was negative for intracranial hemorrhage, mass effects, vasogenic edema or midline drift. A brain MRI was notable for thick nonspecific dural enhancement along the left anterior frontal convexity.

After admission, the patient's mental status continued to rapidly decline. A lumbar puncture was performed and cerebrospinal fluid analysis revealed low glucose of $13 \mathrm{mg} / \mathrm{dl}$, increased protein of $256 \mathrm{mg} / \mathrm{dl}$, chloride of $115 \mathrm{mg} / \mathrm{dl}$, and a $\mathrm{pH}$ of 7.97. Red cell count was 155 cells/ $\mu \mathrm{l}$, and white cell count was 14 cells $/ \mu \mathrm{l}$, with a differential of $14 \%$ neutrophils and $68 \%$ lymphocytes. A cryptococcal antigen test of the CSF fluid was nonreactive and cytology results of the CSF fluid were non-diagnostic. The clinical picture was suggestive of a diagnosis of leptomeningeal carcinomatosis.

Neuro-oncology was consulted regarding the patient's potential for intrathecal chemotherapy treatment. Unfortunately, her condition was deteriorating so rapidly that the family opted for palliative care management, and the patient expired soon afterwards in the hospital.

\section{Discussion}

Carcinomatosis is a condition in which cancer spreads diffusely throughout the body, and can take place in both the peritoneum and the leptomeninges. Leptomeningeal carcinomatosis (LC), also known as neoplastic meningitis, occurs when a patient's cancer metastasizes to the meninges surrounding the spinal cord and spreads diffusely throughout the subarachnoid space to produce multifocal neurological signs and symptoms. It is an end-stage cancer phenomenon, and a relatively rare metastasis.

Malignant cells can metastasize to the meninges in several ways. Hematogenous spread is the most common route, but this type of entry is more often seen in hematologic malignancies like leukemia than in solid primary cancers. Solid tumors tend to spread through lymphatics to reach the meninges, and enter the subarachnoid space by passing through the dural and arachnoid coverings of spinal and cranial nerve roots. Other pathways described include direct spread from metastases in the CNS parenchyma, as well as iatrogenic spread through an accidental ependymal or dural breach created by a neurosurgical procedure. No matter the route, once malignant cells gain access to the CSF, they seed the meningeal surface by CSF flow and grow to form deposits that can appear either diffusely thin, or plaque-like and 
forming nodules along the meninges. The cancerous cells can then invade the pial membrane to reach the spinal nerves, cranial nerves, and spinal cord, resulting in the multifocal neurologic symptoms that characterize the metastasis.

LC is diagnosed clinically in $5-8 \%$ of solid tumor cancer patients and in $5-15 \%$ of patients with leukemia/lymphoma. However, autopsy results of cancer patients who showed neurological signs and symptoms prior to death have revealed undiagnosed LC in $20 \%$ of cases. ${ }^{1}$ This can be explained by the fact that LC is usually an end-stage complication of metastatic cancer; confusion and pain are common in terminal cancer patients, so many cases of symptomatic LC may be overlooked in the late stages of metastatic cancer. ${ }^{2}$

The most common causes of LC are breast and lung cancer, even though other rare malignancies may be more inclined to metastasize to the meninges; this is because breast cancer patients have a longer life expectancy and a larger patient population. Incidences of LC in different cancer populations are as follows: melanoma $>23 \%$, small cell lung cancer $6-25 \%$, leukemias $10 \%$, lymphomas $7 \%$, breast cancer $2-5 \%$, and non-small cell lung cancers $1-5 \% .^{3}$

It has been noted recently that the incidence of LC has been increasing in both the breast and lung cancer populations. ${ }^{2}$ This phenomenon can be explained by several causes. Ongoing improvements in cancer treatment have led to better systemic control of cancer and longer patient survival time, which allows for LC to occur in the end stages of the disease. In addition, many popular chemotherapeutic drugs will not cross the blood-CSF barrier; thus, while the patient is being treated systemically, any subarachnoid tumor cells present are not adequately treated and can still proliferate. Two examples of such chemotherapeutic agents are taxanes and trastuzumab, both of which are commonly utilized in breast and lung cancer. Finally, there is an increased rate of clinical diagnosis of LC due to heightened physician awareness and neuro-imaging studies that can help to confirm the suspicion. ${ }^{2}$

Multifocal neurological symptoms are the classic presentation of LC, but clinical signs can be absent in at least $25 \%$ of patients at time of diagnosis. ${ }^{1}$ Patients most commonly complain of headache, change in mental status, confusion, facial and auditory deficits, diplopia, back pain, and lower extremity numbness or weakness. $15 \%$ of patients will show signs and symptoms associated with cerebral hemispheric dysfunction (headache, dizziness, nausea/vomiting), 35\% of patients will show signs and symptoms associated with cranial nerves (diplopia, facial nerve palsies), and $60 \%$ of patients will show signs and symptoms associated with the spinal cord and nerve roots (radicular pain or weakness, urinary incontinence or retention). ${ }^{1}$

Early diagnosis of LC is essential to prevent further complications and neurological damage, and so all patients suspected of having the disease should receive a lumbar puncture and an MRI of the spinal cord. Classic CSF findings in LC include elevated protein concentration, lymphocytic pleocytosis, and decreased glucose concentration, which were all seen in our patient. MRI findings suggestive of LC include cranial nerve, subependymal, and leptomeningeal enhancement. While CSF cytology with evidence of malignant cells remains the gold standard for diagnosis, cytology results are positive upon first lumbar puncture in only $54 \%$ of LC patients, and may remain falsely negaive in up to $14 \%$ of LC patients after even three CSF samplings. ${ }^{4}$ Cytology is more likely to prove the existence of LC when meningeal involvement is diffuse or when the sampling site is closer to the site of the main focus of metastasis. Even if cytology of the CSF is non-diagnostic, and there exists an opening pressure above $15 \mathrm{~cm} \mathrm{H} 2 \mathrm{O}$, an elevated white blood cell count, a protein concentration greater than $50 \mathrm{mg} / \mathrm{dL}$, or a glucose concentration less than $60 \mathrm{mg} / \mathrm{dL}$, LC remains a likely cause of the patient's symptoms. ${ }^{3}$ Therefore, in advanced cancer patients with multifocal neurological symptoms, typical MRI image findings, and suggestive CSF fluid analysis, the diagnosis of leptomeningeal carcinomatosis is quite straightforward. ${ }^{2}$

Treatment of leptomeningeal carcinomatosis requires a multidisciplinary approach. The current goal of therapy is improvement or stabilization of patients' neurologic symptoms. Therapeutic approaches include intrathecal chemotherapy (mainly with methotrexate), radiotherapy of the meninges, systemic chemotherapy with drugs that penetrate the CSF, and surgery to relieve increased intracranial pressure. ${ }^{5} \mathrm{~A}$ recent study has shown that systemic chemotherapy, intrathecal chemotherapy, and whole brain radiation all have a positive impact on patient survival, while radiotherapy of the spinal meninges does not influence patient survival time. While radiotherapy and intrathecal chemotherapy were once the mainstays of LC treatment, systemic chemotherapy (utilizing drugs that can penetrate the subarachnoid space) is gaining popularity as a therapeutic option, as it treats both the meningeal carcinomatosis as well as the systemic malignancy. Recent recommendations for the treatment of LC suggest focal radiation first to debulk any tumors, followed by a course of chemotherapy. Solid tumors have a propensity to attach to neural structures in the form of bulky nodules, while hematologic malignancies tend to spread more diffusely along the meninges. The leptomeningeal nodules caused by the spread of solid tumors are more likely to show up as areas of enhancement on MRI. Intravenous systemic chemotherapy is preferred in patients who have multiple nodules or MRI evidence of leptomeningeal enhancement, and intrathecal chemotherapy is preferred in patients with positive CSF cytology but negative MRI scans. ${ }^{5}$

Aggressive treatment is unlikely to improve neurologic symptoms in many patients; indeed, neurologic toxicity can result, instead. In these patients, palliation may be the best option. If left untreated, the median survival time of LC patients is 3-6 weeks. With treatment, median survival time is variable, ranging from 7-16.5 weeks depending on the patient's performance status and primary tumor site. ${ }^{3}$ Of the LC patients who die, $24-34 \%$ die from progression of the leptomeningeal 
carcinomatosis itself, $22-25 \%$ die from progression of both LC and the systemic disease, and $19-44 \%$ die from progression of the systemic cancer alone. ${ }^{3}$ Several prognostic factors have been indicated in response to treatment, including age, tumor type, and neurological status at time of diagnosis. A series of studies of LC patients demonstrated that $61 \%$ of breast cancer patients showed a neurological stabilization or advancement with radiation and chemotherapy, while only $39 \%$ of lung carcinoma and $18 \%$ of melanoma patients achieved similar results. Patients with breast cancer as their primary malignancy have been shown to have the best prognosis of all patients with leptomeningeal metastases, and are consequently most likely to benefit from aggressive treatment. ${ }^{2}$

\section{References}

1. Beauchesne P. Intrathecal chemotherapy for treatment of leptomeningeal dissemination of metastatic tumours. Lancet Oncol;11:871-9.

2. Taillibert S, Laigle-Donadey F, Chodkiewicz C, Sanson M, Hoang-Xuan K, Delattre JY. Leptomeningeal metastases from solid malignancy: a review. J Neurooncol 2005;75:85-99.

3. Mammoser AG, Groves MD. Biology and therapy of neoplastic meningitis. Curr Oncol Rep;12:41-9.

4. Clarke JL, Perez HR, Jacks LM, Panageas KS, Deangelis LM. Leptomeningeal metastases in the MRI era. Neurology;74:1449-54.

5. Rudnicka H, Niwinska A, Murawska M. Breast cancer leptomeningeal metastasis-the role of multimodality treatment. J Neurooncol 2007;84:57-62.

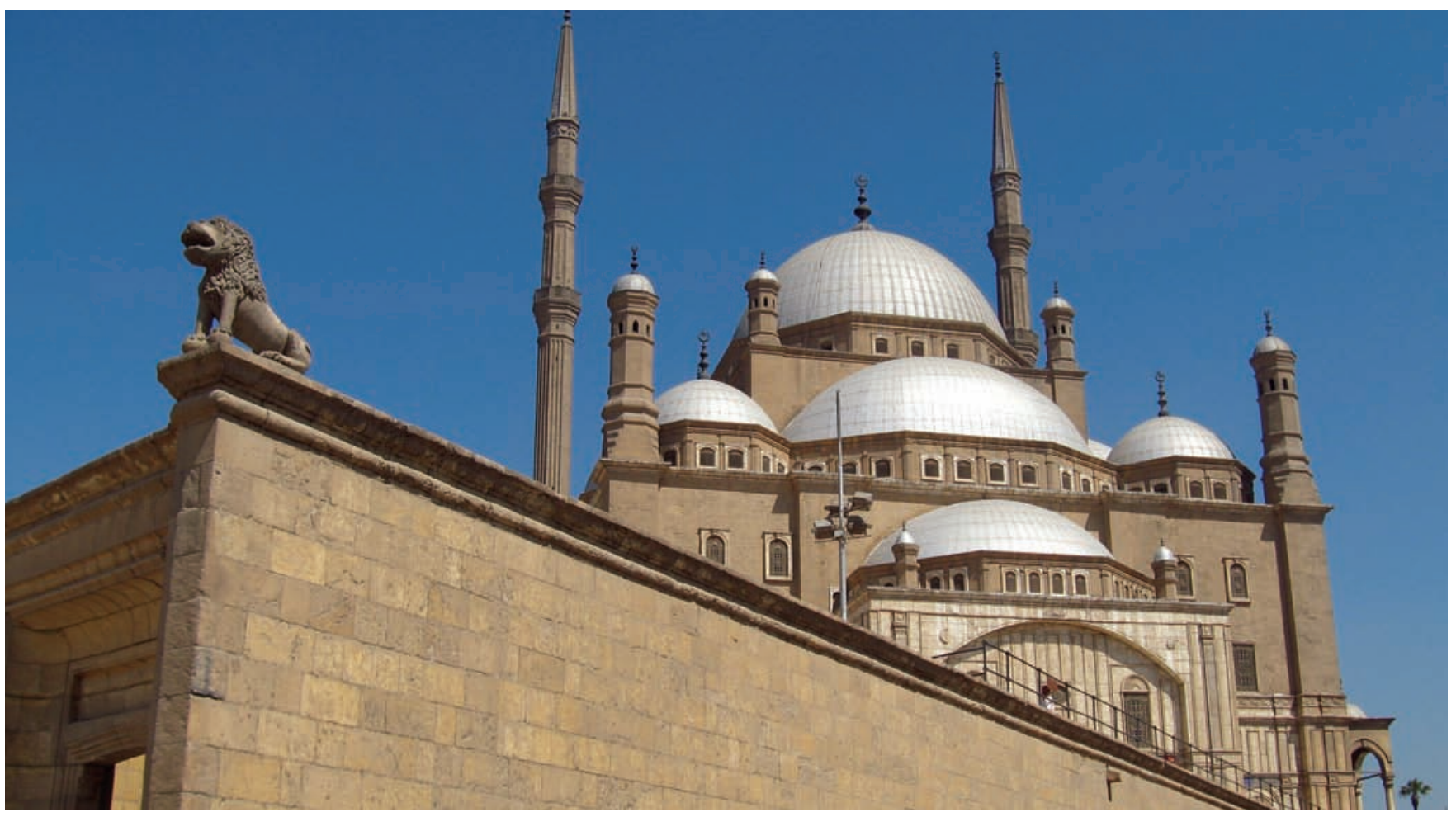

"Citadel of Cairo, Egypt", photograph by Sameh Gaballa, MD 